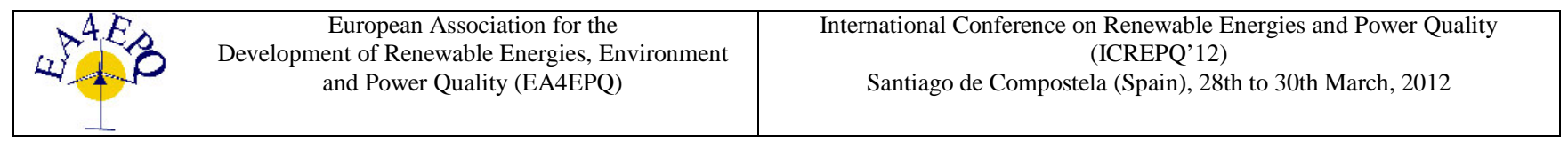

\title{
Impedance-Based Methods for Detection of Voltage Sag Sources
}

\author{
Boštjan Polajžer, Gorazd Štumberger and Drago Dolinar \\ University of Maribor \\ Faculty of Electrical Engineering and Computer Science \\ Smetanova 17, 2000 Maribor, Slovenia \\ phone:+386 2220 7076, fax:+386 2220 7272, e-mail: bostjan.polajzer@uni-mb.si
}

\begin{abstract}
The research reflects impedance-based methods for detection of voltage sag sources, whereas measured voltages and currents are treated as average functions, and also as instantaneous values. Evaluation of the discussed methods has been performed by applying extensive numerical simulations and field testing. The obtained results show that all methods are very effective in the cases of symmetrical voltage sags. When instantaneous values are applied the best effectiveness is obtained also for asymmetrical voltage sags.
\end{abstract}

Key words power systems, power quality, voltage sag, source detection.

\section{Introduction}

Voltage sags (dips) are a decrease in the supply rms voltage between $90 \%$ and $1 \%$ of the declared value, and with duration of mostly less than $1 \mathrm{~s}$. They can be caused by different events related to the network [1]. The majority of voltage sags occur due to the faults of the power system. In cases of two-phase or single-phase faults, which are the mostly frequent, the resulting voltage sags are asymmetrical. The duration of these voltage sags can be as short as three to four cycles and it is associated with the fault clearing time. Due to high values of the magnetizing (inrush) currents, the energizing of a transformer is another source of voltage sags. These voltage sags are asymmetrical and last between $100 \mathrm{~ms}$ and $500 \mathrm{~ms}$. Voltage sags caused by a heavy motor starting (and loading) last longer; few seconds or several tens of seconds. These voltage sags are symmetrical, because electrical motors are generally balanced loads.

Voltage sags can occur due to the events in the distribution or in the transmission system, or also due to the events that origin from the customer side. The impact of disturbances caused by voltage sags on the production losses have already been reported [2], as well as the severe influence of voltage sags on the behavior of induction machines and three-phase transformers [3], [4]. In such cases, any disputes can be resolved fairly if the voltage sags source is reliably detected.
Several methods for detection of voltage sag sources have already been proposed [5]-[15]. These methods give the information on which side of the monitoring point the voltage sag origin is; i.e. from the upstream side (A) or downstream side (B), as it is shown in Fig. 1. In order to pinpoint the exact location of the voltage sag sources a recording device has to be placed in all points of the common coupling between all responsible parties. This is clearly not possible, therefore different methods for tracking the voltage sag sources have been proposed [16] [19], but an overall acceptable methodology has not been developed yet.

This paper evaluates impedance-based methods for detection of voltage sag sources [8]-[11]. In [8] and [9], two methods are proposed, where the impedance angle and magnitude or the real part of the estimated incremental impedance is used. These methods are both phasor-based and can produce questionable results due to the inherent averaging in the harmonic analysis of the input signals. Therefore, new impedance-based method is proposed by applying instantaneous values [10], where Park's transformation ( $d q$-components) is used. However, calculation of the so- called instantaneous impedance might lead to numerical problems because the divisions by zero can occur. Another method is proposed in [11] in order to overcome this difficulty, where the sign of the first peak is observed in incremental norms of voltage and active current vectors. Note that the instantaneous $\alpha \beta$ Clarke's components are used within this method. All the discussed methods for detection of voltage sag sources have been tested by applying extensive numerical simulations and field testing.

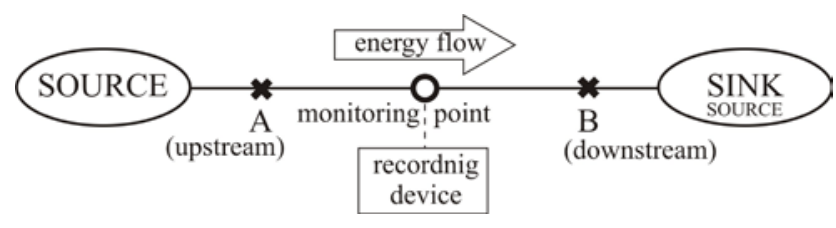

Fig. 1. Upstream event (A) and downstream event (B) 


\section{Brief description of the impedance-based methods}

\section{A. Impedance Magnitude and Angle [8]}

The estimated impedance during the voltage sag changes both with magnitude and in angle. When the estimated impedance magnitude $|\underline{Z}|$ is decreased and the angle $\angle \underline{Z}$ is positive during the voltage sag, the disturbance originates from the downstream side. Otherwise, the voltage sag source is on the upstream side (1), [8].

$$
\begin{aligned}
& \text { if }\left|\underline{Z}_{\text {sag }}\right|<\left|\underline{Z}_{\text {presag }}\right| \text { and } \angle \underline{Z}_{\text {sag }}>0 \quad \Rightarrow \text { downstream } \\
& \text { else } \quad \Rightarrow \text { upstream }
\end{aligned}
$$

\section{B. Incremental Impedance [9]}

Incremental impedance is obtained from the ratio $\Delta \underline{Z}=(\Delta \underline{U} / \Delta \underline{I})$, where the incremental voltage is defined as $\Delta \underline{U}:=\left(\underline{U}_{\text {sag }}-\underline{U}_{\text {presag }}\right)$, and the incremental current is defined as $\Delta \underline{I}:=\left(\underline{I}_{\mathrm{sag}}-\underline{I}_{\text {presag }}\right)$. Note that the phasors are applied for the positive-sequence component, whereas only the real part of the estimated incremental impedance is used as the criterion (2), [9]. It is negative for downstream events and positive for upstream events. To achieve the improvement of the estimated impedance, adaptive multiple cycles of data are used by applying the least-squares method [9].

$$
\operatorname{Re}(\Delta \underline{Z})=\Delta R\left\{\begin{array}{l}
>0 \Rightarrow \text { upstream } \\
<0 \Rightarrow \text { downstream }
\end{array}\right.
$$

\section{Instantaneous Impedance in dq Components [10]}

Instantaneous values are used within this method, whereas $d q$-Park transformation (12), (13) is used. The so- called instantaneous impedance $z_{d q}(t)$ is obtained from the ratio (3), where the instantaneous $d q$-sequence power and the norm of the voltage vector are obtained from (14) and (15), respectively.

$$
z_{d q}(t):=\frac{\left\|\mathbf{u}_{d q}(t)\right\|^{2}}{p_{d q}(t)}
$$

If during the voltage sag the impedance $z_{d q}(t)$ is decreased, the sag source is on the downstream side, else it is on the upstream side (4), [10].

$$
\text { if } \begin{aligned}
z_{d q}(t)_{\text {sag }}<z_{d q}(t)_{\text {presag }} & \Rightarrow \text { downstream } \\
\text { else } & \Rightarrow \text { upstream }
\end{aligned}
$$

\section{Incremental Norms of Voltage and Active Current Vectors in $\alpha \beta$ Components [11]}

Another method is proposed where instantaneous $\alpha \beta$ Clarke's transformation (7), (8) is used. The incremental equivalent resistance is introduced by the ratio (5), where the incremental norm of the voltage vector is defined as $\Delta\left\|\mathbf{u}_{\alpha \beta}(t)\right\|=\left(\left\|\mathbf{u}_{\alpha \beta}(t)\right\|_{\text {sag }}-\left\|\mathbf{u}_{\alpha \beta}(t)\right\|_{\text {presag }}\right)$. The incremental norm of the active current vector is defined as $\Delta\left(S_{\alpha \beta}\left\|\mathbf{i}_{\mathrm{a} \alpha \beta}(t)\right\|\right)=\left(\left(S_{\alpha \beta}\left\|\mathbf{i}_{\mathrm{a} \alpha \beta}(t)\right\|_{\text {sag }}\right)-\left(S_{\alpha \beta}\left\|\mathbf{i}_{\mathrm{a} \alpha \beta}(t)\right\|_{\text {presag }}\right)\right)$, where $S_{\alpha \beta}=\operatorname{sign}\left(p_{\alpha \beta}(t)\right)$. The instantaneous $\alpha \beta$-sequence power and norms of voltage and active current vector are obtained from (9)-(11), respectively.

$$
\Delta R_{\mathrm{e} \alpha \beta}(t):=\frac{\Delta\left\|\mathbf{u}_{\alpha \beta}(t)\right\|}{\Delta\left(S_{\alpha \beta}\left\|\mathbf{i}_{\mathrm{a}, \alpha \beta}(t)\right\|\right)}
$$

The incremental equivalent resistance $\Delta R_{\mathrm{e} \alpha \beta}(t)$ is negative for downstream events and positive for upstream events. Note that computation of the ratio (5) might lead to numerical problems because the divisions by zero can occur. However, only the sign of the ratio is needed for the voltage-sag source-detection criterion. The estimated sign is positive, when the signs of the numerator and denominator are equal, that is for upstream events, otherwise it is negative (downstream event). Thus, the instantaneous resistance- sign based method is proposed by (6), [11].

$$
\frac{\operatorname{sign}\left(\text { first peak }\left(\Delta\left\|\mathbf{u}_{\alpha \beta}(t)\right\|\right)\right)}{\operatorname{sign}\left(\text { first peak }\left(\Delta\left(S_{\alpha \beta}\left\|\mathbf{i}_{\mathrm{a} \alpha \beta}(t)\right\|\right)\right)\right)}\left\{\begin{array}{l}
>0 \Rightarrow \text { upstream } \\
<0 \Rightarrow \text { downstream }
\end{array}\right.
$$

\section{Results}

Let us first consider the network shown in Fig. 2 during the upstream phase-to-phase fault, where voltages and currents are captured in MP2. When methods (1), [8] and (2), [9] are used the obtained impedance is positive $(+1.6 \Omega)$ indeed, but it does not change with respect to time. Because the value of the voltage-current ratio does not change with respect to time, the identical result is obtained even when using the method, which is based on instantaneous impedance (4), [10]. On the other hand, the method (6), [11], gives a conclusive and correct result. As it can be seen in Fig. 3, signs of first peaks in the incremental voltage vector norm and the incremental active current vector norm are equal, which correctly indicates an upstream event.

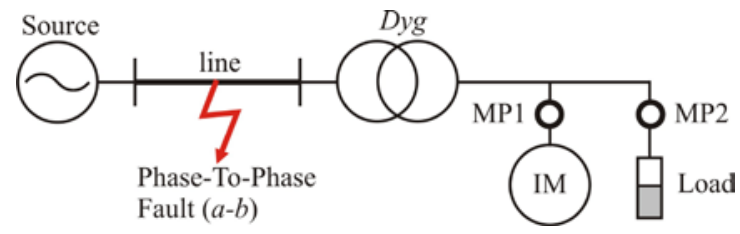

Fig. 2. Radial network during upstream phase $a$ to phase $b$ fault
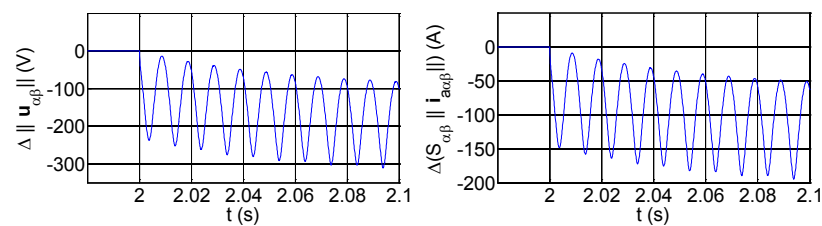

Fig. 3. Incremental norms of voltage and active current vectors for example shown in Fig. 2 


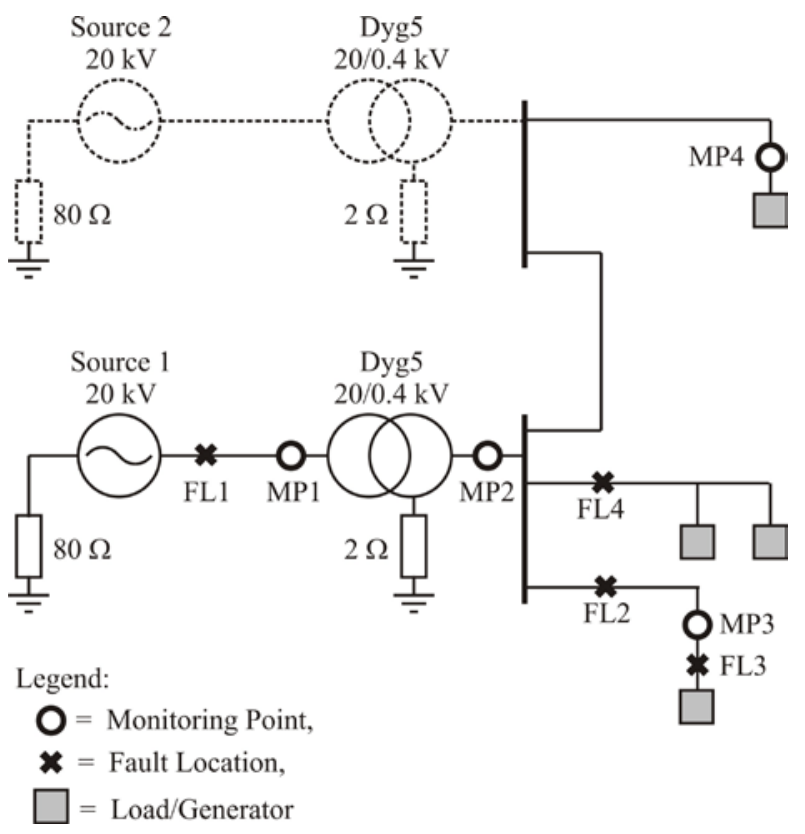

Fig. 4. Testing-network for simulations of voltage sags: radial network (solid line) and non-radial network (solid and dashed line)

\section{A. Numerical Simulations and Field Testing}

A test network (Fig. 4) has been used for numerical simulations of voltage sags. An extensive number of numerical simulations have been performed using MATLAB/Simulink/SimPowerSystems. Different types of loads, such as RL-load, induction and synchronous motors and generators, as well as constant power loads are used in different combinations. Four types of faults are applied in four different locations (FL1-FL4): phase-toearth (P-E), phase-to-phase-to-earth (P-P-E), phase-tophase (P-P) and three-phase (3-P). Furthermore, voltage sags due to the heavy motor starting and loading are also simulated. Voltages and currents are captured in four monitoring points (MP1-MP4).

An extensive number of field tests are also applied to all the discussed methods. Voltages and currents are captured in Slovenian power system for different voltage levels $(400 \mathrm{kV}, 220 \mathrm{kV}, 110 \mathrm{kV}, 20 \mathrm{kV}$ and $0.4 \mathrm{kV})$ with a sampling frequency of $5 \mathrm{kHz}$. The discussed voltage sags are caused by different events, such as 3-P faults, P-P faults, P-E faults and energizing of a transformer.

An example of the voltage sag during the transformer switch on is shown in Fig. 5. As it can be seen from the measured voltage and current waveforms, asymmetrical voltage sag with a magnitude of $\sim 20 \%$ occurs due to the high values of the inrush currents (Figs. $5 \mathrm{a}$ and $5 \mathrm{~b}$ ). After approximately two cycles, extremely asymmetrical voltage sag is caused due to the unknown P-P fault, which was quickly developed into a 3-P fault. In order to determine the side on which these two events origin, all the discussed methods for detection of voltage sag sources are used. As it can be seen in Figs. $5 c$ and $5 d$ the magnitude of the calculated impedance decreases during the sag, while its angle remains positive. This result indicates a downstream event, yet due to a considerable time delay this result might be questionable. The result obtained using the real part of the incremental impedance is inconclusive, because the calculated value is zero $(\operatorname{Re}(\Delta \underline{Z})=0)$. The impedance calculated with instantaneous $d q$-Park's components is shown in Fig. 5e, where severe spikes can be observed due to the divisions with small numbers. Such result cannot be interpreted in a unique way. Fig. $5 f$ shows time responses for incremental norms of voltage and active current vectors in $\alpha \beta$-Clarke's components. Because their signs are being opposite a downstream event is indicated.
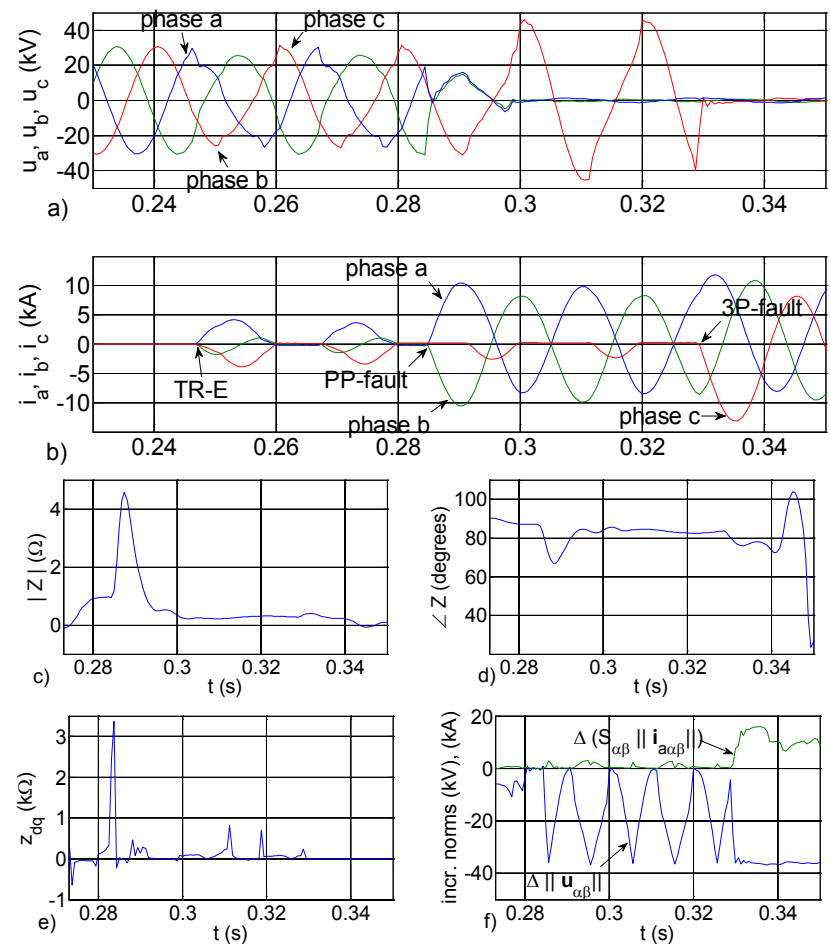

Fig. 5. Measured voltage and current waveforms (a, b) for the P-P and 3 $\mathrm{P}$ fault during the energizing of the transformer, and obtained results using all the discussed methods (c, d, e, f)

\section{B. Evaluation of the Impedance-Based Methods}

Evaluation of the discussed methods for detection of voltage sag sources is performed by applying extensive numerical simulations of voltage sags. Effectiveness is estimated for all the discussed methods. The results presented in Table I show that the effectiveness of methods (1), [8] and (2), [9] is quite low, whereas the results obtained using instantaneous $d q$-sequence impedance (4), [10] are questionable. The results obtained for the instantaneous resistance- sign based method (6), [11] show the very high effectiveness.

TABLE I

EFFECTIVENESS OF DISCUSSED METHODS

\begin{tabular}{|c|c|c|c|c|c|}
\hline method & $\begin{array}{l}\text { criterion } \\
\text { (eq.), [ref.] }\end{array}$ & $\begin{array}{l}\text { symmetrical } \\
\text { sags }\end{array}$ & $\begin{array}{l}\text { asymmetrical } \\
\text { sags }\end{array}$ & $\begin{array}{l}\text { earth } \\
\text { faults }\end{array}$ & total \\
\hline$|\underline{Z}|, \angle \underline{Z}$ & $(1),[8]$ & $\bullet$ & ? & ?? & $\bullet$ \\
\hline $\operatorname{Re}(\Delta \underline{Z})$ & (2), [9] & $\bullet$ & ?? & ?? & $\bullet$ \\
\hline$z_{d q}(t)$ & (4), [10] & - & - & - & ?? \\
\hline$\Delta R_{\mathrm{e} \alpha \beta}(t)$ & (6), [11] & $\bullet \bullet$ & $\bullet \bullet$ & $\bullet \bullet$ & $\bullet \bullet$ \\
\hline
\end{tabular}

Effectiveness: •• very high (> 95\%), • acceptable (75\% - 95\%),

?? questionable (50\% - 75\%), - insufficient (<50\%). 


\section{Conclusion}

This research gives a general description of impedancebased methods for detection of voltage sag sources. An overall evaluation of all discussed methods is performed throughout the extensive numerical simulations and field testing. On the basis of the obtained results, it can be concluded that all the methods can be used in the cases of symmetrical voltage sags. In cases of asymmetrical voltage sags, the method, which is based on the instantaneous $\alpha \beta$-Clarke's components is the most effective one.

\section{Acknowledgement}

This work has been supported by ARRS, Projects no. P20115 and L2-2060.

\section{References}

[1] M. J. H. Bollen, Understanding power quality problems: voltage sags and interruptions (IEEE Press, New York, 2000).

[2] C. J. Melhorn, T. D. Davis and G. E. Beam, "Voltage sags: their impact on the utility and industrial customers," IEEE Trans. Ind. Appl., vol. 34, no. 3, pp. 549-558, 1998.

[3] L. Guasch, F. Córcoles, J. Pedra, Effects of symmetrical and unsymmetrical voltage sags on induction machines, IEEE Trans. Power Del., vol. 19, no. 2, pp. 774-782, 2004.

[4] J. Pedra, L. Sáinz, F. Córcoles, L. Guasch, Symmetrical and unsymmetrical voltage sag effects on three-phase transformers, IEEE Trans. Power Del., vol. 20, no. 2, pp. 1683-1691, 2005.

[5] A. C. Parsons, W. M. Grady, E. J. Powers and J. C. Soward, A direction finder for power quality disturbances based upon disturbance power and energy, IEEE Trans. Power Del., vol. 15, no. 3, pp. 1081-1086, 2000.

[6] C. Li, T. Tayjasanant, W. Xu and X. Liu, Method for voltage-sagsource detection by investigating slope of the system trajectory, IEE Proc. Gen. Trans. Distrib., vol. 150, no. 3, pp. 367-372, 2003.

[7] N. Hamzah, A. Mohamed, A. Hussain, A new approach to locate the voltage sag source using real current component, Electric Power Syst. Research, vol. 72, no. 2, pp. 113-123, 2004.

[8] A.K. Pradhan, A. Routray, Applying Distance Relay for Voltage Sag Source Detection, IEEE Trans. Power Del., vol. 20, no. 1, pp. 529-531, 2005.

[9] T. Tayjasanant,V. Li, and W. Xu, A resistance sign-based method for voltage sag source detection, IEEE Trans Power Del., vol. 20, no. 4, pp. 2544-2551, 2005.

[10] T. Shao, J.-P. Peng, J. Kang, Locating Voltage Sag Source with Impedance Measurement, Int. Conf. on Power Syst. Technology POWERCON 2010 , Hangzhou, China, October 24-28, 2010.

[11] B. Polajžer, G. Štumberger, S. Seme, D. Dolinar, Generalization of Methods for Voltage Sag Source Detection Using Vector Space Approach, IEEE Trans. Ind. Appl., vol. 45, no. 6, pp. 2152-2161, 2009.

[12] A.K. Pradhan, A. Routray, S.M. Gudipalli, Fault Direction Estimation in Radial Distribution System Using Phase Change in Sequence Current, IEEE Tran. Power Del., vol. 22, no. 4, pp. 2065-2071, 2007.

[13] Z. Galijasevic, A. Abur, Fault Location Using Voltage Measurements, IEEE Trans. Power Del., vol. 17, no. 4, pp. 441445, 2002.

[14] J. Gomes, M. Morcos, D. Tourn, M. Felici, A Novel Methodology to Locate Originating Points of Voltage Sags in Electric Power Systems, $18^{\text {th }}$ Int. Conf. on Electricity Distribution $\sim$ CIRED 2005 , Torino, Italy, June 6-9, 2005.

[15] S.-J. Ahn, D.-J. Won, I.-Y. Chung, D.-I. Moon, Determination of Relative Location of Voltage Sag Source According to Event Cause, IEEE PES General Meeting, Denver, USA, June 6-10, 2004.

[16] G.W. Chang, J.-P. Chao, H.M. Huang, C.-I. Chen, S.-Y. Chu, On Tracking the Source Location of Voltage Sags and Utility Shunt Capacitor Switching Transients, IEEE Tran. Power Del., vol. 23, no. 4, pp. 2124-2131, October 2008.
[17] A. Latheef, M. Negnevitsky, V. Faybisovich, Voltage Sag Source Location Identification, $20^{\text {th }}$ Int. Conf. on Electric Distribution, C CIRED 2009 , Prague, Czech Republic, June 8-11, 2009.

[18] H. Liao, Voltage Sag Source Location in High-Voltage Power Transmission Networks, General Meeting of the IEEE Power and Energy Society, Pittsburgh, USA, July 20-24, 2008.

[19] A. Khosravi, J. Melendez, J. Colomer, J. Sanchez, A Hybrid Method for Sag Source Location in Power Network, $9^{\text {th }}$ Int. Conf. Electrical Power Quality and Utilisation , Barcelona, Spain, October 9-11, 2007.

\section{Appendix}

\section{Clarke's Transformation}

The instantaneous line voltages $u_{a}(t), u_{b}(t), u_{c}(t)$ and currents $i_{a}(t), i_{b}(t), i_{c}(t)$ are transformed into the voltages $u_{\alpha}(t), u_{\beta}(t), u_{0}(t)$ and currents $i_{\alpha}(t), i_{\beta}(t), i_{0}(t)$ by $(7)$, where C denotes the orthogonal Clarke's transformation matrix (8).

$$
\begin{aligned}
& {\left[\begin{array}{l}
u_{\alpha}(t) \\
u_{\beta}(t) \\
u_{0}(t)
\end{array}\right]=\mathbf{C}\left[\begin{array}{l}
u_{a}(t) \\
u_{b}(t) \\
u_{c}(t)
\end{array}\right] ; \quad\left[\begin{array}{l}
i_{\alpha}(t) \\
i_{\beta}(t) \\
i_{0}(t)
\end{array}\right]=\mathbf{C}\left[\begin{array}{l}
i_{a}(t) \\
i_{b}(t) \\
i_{c}(t)
\end{array}\right]} \\
& \mathbf{C}=\sqrt{\frac{2}{3}}\left[\begin{array}{ccc}
1 & -\frac{1}{2} & -\frac{1}{2} \\
0 & \frac{\sqrt{3}}{2} & -\frac{\sqrt{3}}{2} \\
\frac{1}{\sqrt{2}} & \frac{1}{\sqrt{2}} & \frac{1}{\sqrt{2}}
\end{array}\right] ; \quad \mathbf{C}^{-1}=\mathbf{C}^{\mathrm{T}}
\end{aligned}
$$

The instantaneous $\alpha \beta$-sequence power is given by (9), and norms of voltage and active current vector are obtained from (10) and (11), respectively.

$$
\begin{aligned}
& p_{\alpha \beta}(t)=u_{\alpha}(t) i_{\alpha}(t)+u_{\beta}(t) i_{\beta}(t) \\
& \left\|\mathbf{u}_{\alpha \beta}(t)\right\|=\sqrt{u_{\alpha}^{2}(t)+u_{\beta}^{2}(t)} \\
& \left\|\mathbf{i}_{\mathrm{a} \alpha \beta}(t)\right\|=\frac{\left|p_{\alpha \beta}(t)\right|}{\left\|\mathbf{u}_{\alpha \beta}(t)\right\|}
\end{aligned}
$$

\section{Park's Transformation}

The instantaneous voltages $u_{\alpha}(t), u_{\beta}(t)$ and currents $i_{\alpha}(t)$, $i_{\beta}(t)$ are further transformed into the voltages $u_{d}(t), u_{q}(t)$ and currents $i_{d}(t), i_{q}(t)$ by (12), where $\mathbf{P}$ denotes the orthogonal Parks's transformation matrix (13), and $\omega_{0}$ is the nominal frequency.

$$
\begin{array}{ll}
{\left[\begin{array}{l}
u_{d}(t) \\
u_{q}(t)
\end{array}\right]=\mathbf{P}\left[\begin{array}{l}
u_{\alpha}(t) \\
u_{\beta}(t)
\end{array}\right] ;} & {\left[\begin{array}{l}
i_{d}(t) \\
i_{q}(t)
\end{array}\right]=\mathbf{P}\left[\begin{array}{l}
i_{\alpha}(t) \\
i_{\beta}(t)
\end{array}\right]} \\
\mathbf{P}=\left[\begin{array}{cc}
\cos \left(\omega_{0} t\right) & \sin \left(\omega_{0} t\right) \\
-\sin \left(\omega_{0} t\right) & \cos \left(\omega_{0} t\right)
\end{array}\right] ; & \mathbf{P}^{-1}=\mathbf{P}^{\mathrm{T}}
\end{array}
$$

The instantaneous $d q$-sequence power is given by (14), and norm of the voltage vector is obtained from (15).

$$
\begin{aligned}
& p_{d q}(t)=u_{d}(t) i_{d}(t)+u_{q}(t) i_{q}(t) \\
& \left\|\mathbf{u}_{d q}(t)\right\|=\sqrt{u_{d}^{2}(t)+u_{q}^{2}(t)}
\end{aligned}
$$

